\title{
A Gas Outburst Prediction Model Based on Data Mining and Information Fusion
}

\author{
$\operatorname{Min} \mathrm{He}^{1,2}$ \\ ${ }^{1}$ CCTEG Changzhou Research Institute, Changzhou 213015, China \\ ${ }^{2}$ Tiandi (Changzhou) Automation Co., Ltd., Changzhou 213015, China
}

Corresponding Author Email: heminccteg@163.com

https://doi.org/10.18280/ria.330508

Received: 1 May 2019

Accepted: 18 August 2019

Keywords:
gas outburst, data mining,
backpropagation neural network
(BPNN), improved particle swarm
optimization (IPSO), Dempster-Shafter
$(D-S)$ theory of evidence

\begin{abstract}
The gas outburst in coalmines is influenced by multiple factors. These influencing factors are highly uncertain and have complex nonlinear relationships. Considering these features, this paper puts forward a gas outburst prediction model based on data mining and information fusion. On the feature level, the backpropagation neural network (BPNN) was selected to set up a gas outburst identification model, thanks to its strong self-learning ability, and then optimized by the improved particle swarm optimization (IPSO); then, the outputs of the optimized BPNN were taken as the identification results, and used to establish a feature database. On the decision level, the Dempster-Shafter (D-S) theory of evidence was introduced to fuse the identification results in the time domain and the spatial domain, and make decisions on the gas state of the coalmine based on the fused data. Finally, the proposed model was applied the predict the gas outburst in a coalmining area of a coalmine in Shanxi Province, China, using the data collected from the workface, intake airway, return airway and transport roadway. Our model fuses the data on two layers, namely, the time domain and the spatial domain, and reduces the prediction uncertainty to zero. The results show that our model can optimize the prediction parameters, enhance the accuracy of gas monitoring information, and improve the correctness of decisions concerning gas outburst in the coalmine.
\end{abstract}

\section{INTRODUCTION}

China is a world-leading coal producer. Roughly $30 \%$ of its coalmines either have a high gas content or face a high risk of gas outburst. In China, gas outburst accidents occur from time to time, posing a serious threat to work safety of coalmines [1]. Gas outburst refers to the sudden and violent ejection of coal and gas from the coal seam (rock mass). It is an extremely dangerous dynamic phenomenon in the coalmining process. A gas outburst often brings heavy casualties and property losses, and may induce secondary incidents like fire and gas explosion $[2,3]$.

As coalmines get deeper, the risk and damage of gas outburst are soaring, under the high geo-stress and high gas pressure. Being a complex dynamic phenomenon in coal mining, gas outburst is formed under the action of multiple factors. Thus, the data on gas outburst are a collection of numerous parameters, various objectives and intricate information. Currently, there is a huge amount of data on gas outburst. However, the data are inaccurate, difficult to model, and dynamically changing. To predict gas outburst and ensure coalmine safety, it is imperative to extract valuable information out of the massive data. Many experts and scholars have explored deep into gas outburst, and proposed many hypotheses on its formation mechanism. Some hypotheses attribute the gas outburst to the action of a single factor, and some ascribe it to the action of multiple factors [4$6]$.

The single-factor hypotheses emphasize on the dominant role of a single factor in the formation of gas outburst, such as gas, geo-stress and chemical reactions. The multi-factor hypotheses consider gas outburst the result of combined effects of stress, gas pressure and physical-mechanical properties of coal. Most scholars at home and abroad have accepted the multi-factor hypotheses, because these hypotheses consider both the forces and media at the gas outburst. As a result, a series of indices about stress, gas pressure or the physical-mechanical properties of coal have been adopted for the prediction of gas outburst, giving birth to many effective prediction methods. The most popular methods fall into comprehensive index prediction (CIP) and noncontact prediction (NCP).

The CIP boasts excellent prediction results. This prediction strategy makes use of indices like gas pressure, gas content, geo-stress, coal seam thickness, firmness coefficient of coal and damage type of coal. Many advanced mathematical approaches have been integrated into the CIP, namely, neural network (NN), fuzzy comprehensive evaluation (FCE), gray system theory (GST), support vector machine (SVM) and data fusion. Meanwhile, the NCP relies on methods like gas emission characteristic method, acoustic emission, electromagnetic radiation and micro-seismic monitoring, and has been applied in many coalmines [7].

\section{LITERATURE REVIEW}

Gas outburst is a dynamic phenomenon induced and influenced by multiple complex factors. For many years, domestic and foreign researchers have extensively explored the mechanism and evolution of gas outburst, and predicted its occurrence, yielding fruitful results [8-18]. For instance, An et 
al. [8] provided a method to calculate the gas expansion energy for outburst initiation, according to the effect of coal damage and the change of environmental pressure for desorption, and applied the method to predict the gas state in coalmines. Inspired by data mining, Nilufer and Mustafa [9] established a gas disaster information fusion model based on fuzzy-rough set data mining algorithm, and thus improved the prediction accuracy of gas outburst. Chen et al. [10] designed a gasdriven gas outburst test system, which can predict the gas outburst for standard coals and raw coals at different temperatures and gas pressures. Liu et al. [11] combined the fuzzy neural network (FNN) and Dempster-Shafter (D-S) theory of evidence to determine the level of gas outburst risk of coalmine workface, took the NN output as the basic belief assignment function, and predicted the gas outburst after fusing information twice.

Data mining and information fusion are two techniques to process massive data and extract useful knowledge. The former mainly extracts the unknown and potentially usable patterns hidden in a large amount of incomplete, noisy, and fuzzy data, while the latter aims to produce new meaningful information through multi-level, multi-faceted and multi-layer processing and combination of the data collected by several groups of sensors [9]. The two techniques complement each other, and can be integrated deeply to analyze complex data in real-world applications. Therefore, it is very meaningful to apply data mining and information fusion to prediction of gas outburst in coalmines.

Tu et al. [12] established an experimental device to simulate the gas outburst of coal seam, and studied the influence of abnormal gas distribution on the outburst. Liu et al. [13] developed a hybrid prediction method based on genetic algorithm (GA) and backpropagation neural network (BPNN), and optimized the weights and thresholds of the NN with the global search ability of the GA. To predict gas outburst, Papalexakis et al. [14] optimized the mainly parameters of the SVM with differential evolution-distributed estimation algorithm, and improved the training speed and accuracy of the prediction model. Tu and Yan [15] created an earlywarning method for gas outburst based on wavelet packet entropy and data fusion, in which the data from multiple sensors are processed through mean-based batch estimation and fusion and the features are extracted according to the number of layers of wavelet packet decomposition based on reconstructed signal energy; the early-warning method was proved to be effective through experiments. To sum up, the above studies have presented rather good methods to predict the gas outburst in coalmines. However, there are still several defects with these methods: First, the NN faces problems like overlearning and slow convergence; the classification effect of SVM models depends on the selection of kernel function and penalty parameters, which are difficult to choose rationally.

According to the above review, the existing prediction strategies for gas outburst cannot always issue correct early warning of gas outburst, for they often overlook how sensitive the outburst indices respond to gas outburst, or manually assign weights to the indices. To solve the problem, this paper fully integrates data mining with information fusion to predict gas outburst in coalmines. Firstly, the BPNN, an NN with selflearning ability, was adopted to construct a gas outburst identification model, and optimized by the improved particle swarm optimization (PSO); the outputs of the optimized BPNN were taken as the identification results, and used to establish a feature database. Next, the D-S theory of evidence was employed to fuse the identification results in the time domain and the spatial domain, and make accurate decisions on the gas state in the coalmine based on the fused data.

\section{METHODOLOGY}

\subsection{Gas outburst prediction model}

The gas outburst is a severe natural disaster that bottlenecks work safety in coalmines. The existing gas monitoring system mainly performs local monitoring and management, such as judging whether the local data have surpassed the limit, and outputting the data collected at the same point. There is a lack of effective processing or interpretation of the monitoring data, making it difficult to predict or prewarn gas outburst [16-18]. To make matters worse, gas outburst is influenced by various factors. It is irrational to make accurate predictions based on a single factor or by a single method. To overcome the above defects, this paper integrates data mining and information fusion to predict gas outburst. The merits of the two techniques were combined to improve the prediction accuracy.

Our gas outburst prediction model was established in two steps. First, on the feature level, the BPNN was selected to set up a gas outburst identification model, thanks to its strong selflearning ability, and then optimized by the IPSO; then, the outputs of the optimized BPNN were taken as the identification results, and used to establish a feature database. Second, on the decision layer, the D-S theory of evidence was employed to fuse the identification results in the time domain and the spatial domain, and make decisions on the gas state of the coalmine based on the fused data.

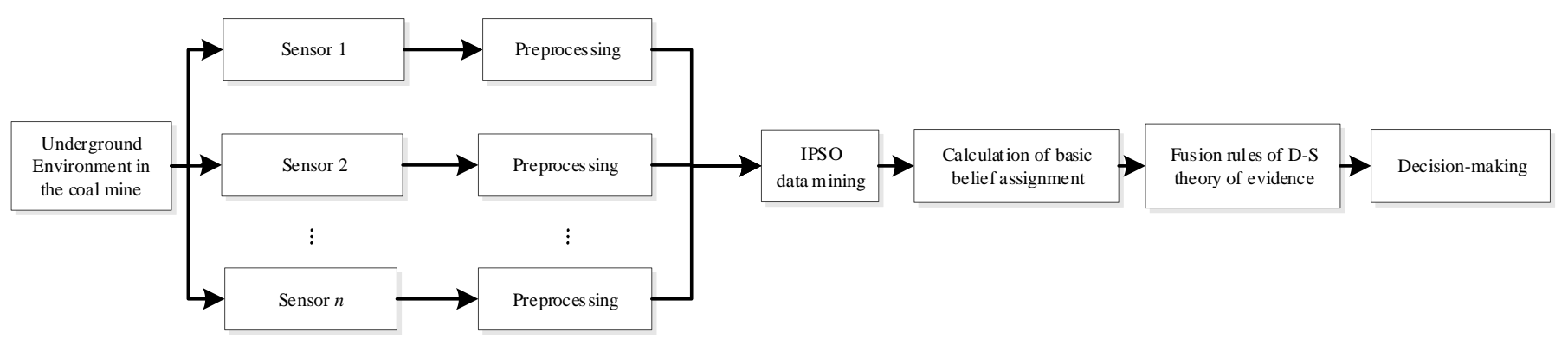

Figure 1. Gas outburst prediction model based on data mining and information fusion

On the feature level, the main operations are data mining and modelling. The raw data were analyzed, and then the features were extracted from the data. Based on normal and abnormal information, a feature database was set up for 
modelling. Several self-learning modelling methods based on data mining are often adopted to learn and recognize the decision-making rules and construct classifiers: rough set theory (RST), decision tree (DT), multilayer perceptron (MLP) and BPNN.

On the decision layer, the decision-making and reasoning were conducted based on the information fusion method and the model established based on data mining. The decisions could be made by the D-S theory of evidence, the RST or NN. The established prediction model for gas outburst in coalmines is as shown in Figure 1 above.

\subsection{Data mining algorithm of gas outburst information}

In the coalmine, the underground environment is extremely complex. Thus, most of the data collected by the gas monitoring system are noisy, incomplete and stochastic. To make accurate prediction of gas outburst, the useful patterns should be fully mined out of the collected data, providing a strong support to risk control and decision-making of gas outburst risk.

There are two types of learning methods for data mining: supervised learning and unsupervised clustering. Supervised learning is to estimate unknown correlations from known input and output samples, establish a classification model, and use the model to classify the instances of unknown sources [19]. The BPNN is a typical supervised learning method. During BPNN-based self-learning modeling, the free parameters can adjust themselves adaptively to the changing environment. However, the BPNN is slow in convergence and prone to the local optimum trap.

Proposed by Eberhart and Kennedy, the PSO is an evolutionary algorithm based on swarm intelligence, which mimics the movement behavior of bird flock or fish swarm. The PSO has been widely adopted to solve optimization problems, because it enjoys a powerful global search ability, adopts the real number code and has a few parameters to be adjusted. It is easy to train any $\mathrm{NN}$ and improve its convergence speed with the PSO [20-22]. Therefore, this paper integrates the PSO and the BPNN, giving full play to the global search ability of the PSO and the local search ability of the BPNN. The integration mainly optimizes the connection weights and topology of the BPNN.

\subsubsection{BPNN}

The BPNN is one of the most popular and mature NNs. This network contains three or more layers of neurons. There is usually an input layer, one or more hidden layers and an output layer [18]. Once a learning sample is inputted to the network, the activation value of each neuron on the input layer will to the output layer via the intermediate hidden layers. Then, the neurons on the output layer will respond to the input. The response will be backpropagated to the input layer via the intermediate hidden layers, with the aim to minimize the error between actual output and desired output. In this way, the connection weights are corrected layer by layer. As the error backpropagation continues, the BPNN can make increasingly correct responses to the inputs. Figure 2 illustrates the structure of a three-layer BPNN.

Let $n, p$ and $q$ be the number of neurons on the input layer, hidden layer and output layer of the BPNN, respectively, $m$ be the number of learning samples, $A^{k}=\left(a_{1}^{k}, a_{2}^{k}, \ldots, a_{n}^{k}\right)(k=1,2, \ldots, m)$ be the input vector and $Y^{k}=\left(y_{1}^{k}, y_{2}^{k}, \ldots, y_{q}^{k}\right)$ be the corresponding output vector. Suppose each hidden layer is activated by
Sigmoid function.

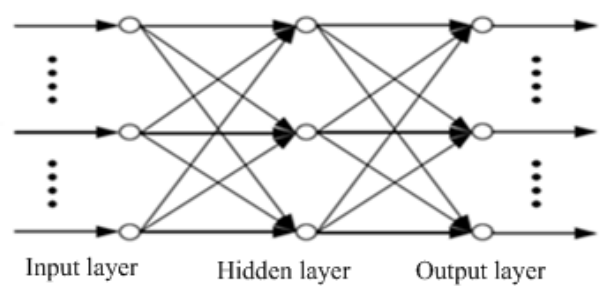

Figure 2. The structure of a three-layer BPNN

Then, the output $b_{j}^{k}$ of hidden layer neuron $j$ can be expressed as:

$$
b_{j}^{k}=f\left(\sum_{j=1}^{n} \omega_{i j} a_{i}^{k}-\theta_{j}\right), \quad j=1,2, \cdots, p
$$

where, $\omega_{i j}$ is the connection weight between input and hidden layers; $\theta_{j}$ is the threshold of the hidden layer.

The output of output layer neuron $t$ can be expressed as:

$$
c_{t}^{k}=f\left(\sum_{j=1}^{n} v_{j t} b_{j}^{k}-\gamma_{t}\right), t=1,2, \cdots, q
$$

where $v_{j t}$ is the connection weight between hidden and output layers; $\gamma_{t}$ is the threshold of the output layer.

The generalized error of each output layer neuron can be expressed as:

$$
d_{t}^{k}=y_{t}^{k}-c_{t}^{k}, t=1,2, \cdots, q
$$

The error function can be established as:

$$
E_{k}=\sum_{t=1}^{q}\left(d_{t}^{k}\right)^{2} / 2
$$

This paper designs a three-layer BPNN containing six neurons on the input layer and five neurons on the output layer. The input layer neurons represent the influencing factors of gas outburst, including the initial velocity of gas diffusion, type of coal failure, gas pressure and geological structure; the output layer neurons represent the existence or severity of abnormality, namely, safe, mild, medium, severe, extremely severe. The selection of hidden layer neurons is the key to the successful simulation of the BPNN. The number of hidden layer neurons directly hinges on the problem requirements, the inputs and the outputs. This number is generally determined empirically or through tests. The weights of the BPNN were adjusted to desirable levels through iterative training of the BPNN by the IPSO

\subsubsection{BPNN optimization by the IPSO}

The PSO is a swarm intelligence algorithm inspired by the migration and aggregation behaviors of a bird flock in the foraging process. The algorithm is simple and easy to realize, and has been widely applied in various fields [23].

Suppose there exists a swarm of $m$ particles in a $D$ dimensional space. Let $x_{i}=\left(x_{i 1}, x_{i 2}, \ldots, x_{i D}\right)^{T}, i=1,2, \ldots, m$ be the position of particle $i, v_{i}=\left(v_{i 1}, v_{i 2}, \ldots, v_{i D}\right)^{T}$ be the velocity of particle $i, p_{i}=\left(p_{i 1}, p_{i 2}, \ldots, p_{i D}\right)^{T}$ is the best-known position of 
particle $i$ at time $t$, and $p_{g}=\left(p_{g 1}, p_{g 2}, \ldots, p_{g D}\right)^{T}$ is the best-known position of the swarm at time $t$. During the optimization in free space, each particle updates its velocity and position by:

$$
\begin{gathered}
v_{i D}(t+1)=\omega v_{i D}(t)+c_{1} r_{1}\left[p_{i D}-x_{i D}(t)\right]+c_{2} r_{2}\left[p_{g D}-x_{i D}(t)\right] \\
x_{i D}(t+1)=x_{i D}(t)+v_{i D}(t+1)
\end{gathered}
$$

where, $\omega$ is the inertia coefficient; $r_{1}$ and $r_{2}$ are random numbers in $[0,1] ; c_{1}$ and $c_{2}$ are learning factors. The intervals of the position and velocity of a particle can be set properly to control its movement.

In the PSO, each particle gradually approaches the individual and global extremes. However, it is very likely that all the particles converge to and stop at an extreme value, resulting in a decline of convergence. To solve the problem, $\mathrm{Xu}$ proposed the sub-dimension (s-d) PSO, which iteratively updates each dimension of a particle and evaluates the fitness in each dimension after the update. By the s-dPSO, the updated position of particle $i$ can be expressed as:

$$
x_{i}^{t^{t}}=x_{i 1}^{(t)}+x_{i 2}^{(t)} \cdots\left(x_{i d}^{(t)}+v_{i d}^{(t+1)}\right) \cdots v_{i m}^{(t+1)}
$$

Then, is compared with $x_{i}^{t}$ in fitness, and the one with the higher fitness is selected $x_{i}^{t}$ for the next iteration. After all the dimensions have been updated, $x_{i}^{(t+l)}=x_{i}^{(t)}$. Since the fitness of each dimension is always improved through the update, the updated value of each particle must be the best-known value since the start of the iterative process. In the IPSO, the velocity of each particle is updated by:

$$
v_{i d}^{(t+1)}=\omega v_{i d}^{(t)}+c_{1} r_{1}\left(p_{g d}^{(t)}-x_{i d}^{(t)}\right)
$$

The degree of particle aggregation in each dimension can be described by the dimension diversity $d(j)$ :

$$
d(j)=\sqrt{\frac{1}{n} \sum_{i=1}^{n}\left(x_{i, j}-\overline{x_{j}}\right)^{2}}
$$

The value of $d(j)$ of a dimension is negatively correlated with how many particles aggregate in that dimension. Then, the particles are sorted by $d(j)$, and the top-ranking particles are subjected to mutation: the value of each particle in that dimension is redefined to disrupt the aggregation of particles.

The BPNN optimized by the IPSO can be implemented in the following steps:

Step 1: Determine the structure and parameters of the BPNN based on the IPSO.

The number of input layer neurons $I$ and the number of output layer neurons $O$ of the BPNN are determined according to the length of input vector and that of output vector, respectively. The number of hidden layer neurons $H$ is selected by experts in an empirical manner. The size of particle swarm $N$, the initial and final inertia weights $\omega_{\text {start }}$ and $\omega_{\text {end }}$, the learning factors $c_{1}$ and $c_{2}$, and the maximum number of iterations $G_{\max }$ are set up in turn. In addition, the values of $\omega_{i j}$, $v_{j k}, \theta_{j}$ and $\psi_{k}$ are randomly initialized within $(0,1)$.

Step 2: Establish the mapping between particles in the IPSO and the parameters to be optimized in the three-layer BPNN. Note that these parameters should be expressed as 1D matrices.

Step 3: Compute the fitness matrix.

A fitness matrix should be established to evaluate the quality of each particle in the swarm. During the training, the sum squared error (SSE) and mean squared error (MSE) between the actual outputs and desired outputs of the BPNN can be respectively computed as:

$$
S S E=\sum_{p=1}^{p} \sum_{k=1}^{0}\left(y_{k}-c_{k}\right)^{2}
$$

$$
M S E=\frac{1}{p} \sum_{p=1}^{p} \sum_{k=1}^{0}\left(y_{k}-c_{k}\right)^{2}
$$

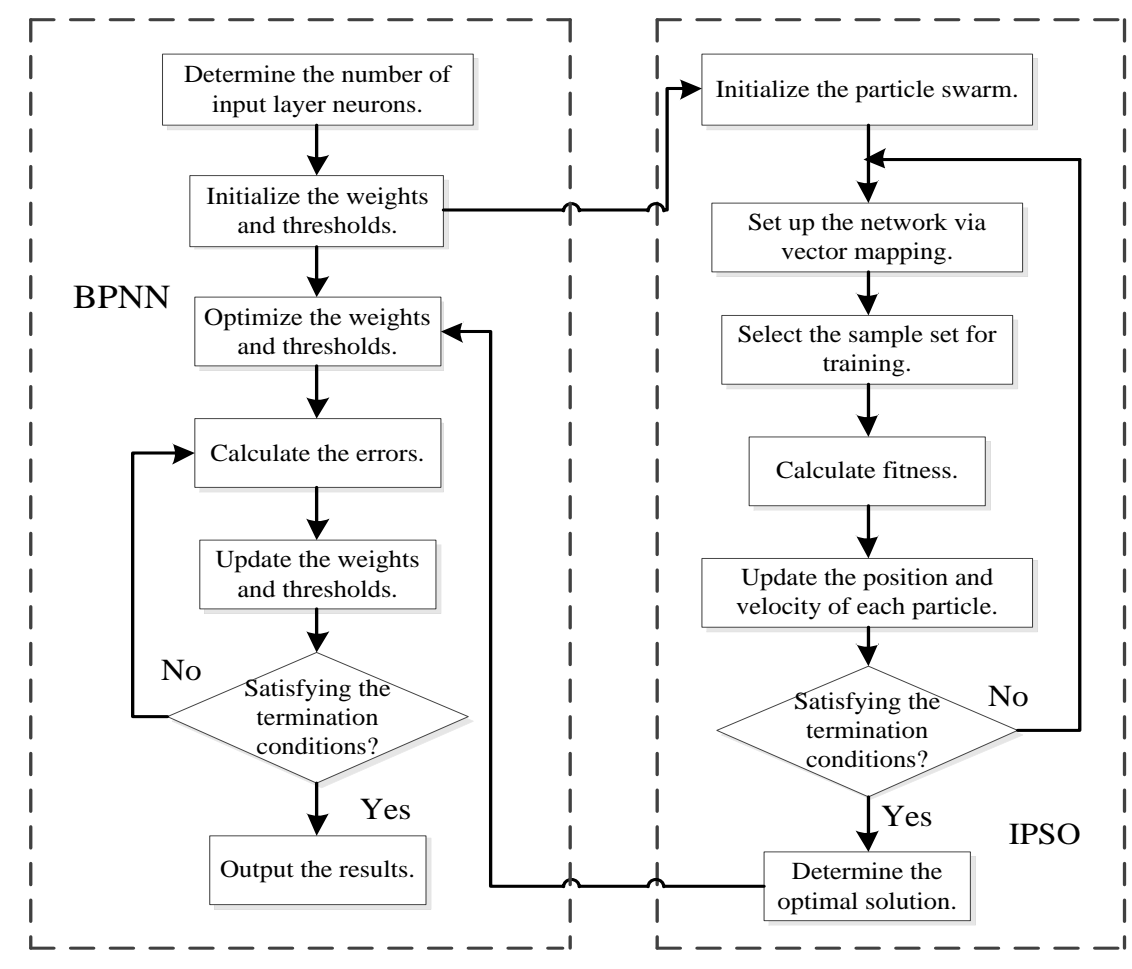

Figure 3. The workflow of the optimization of the BPNN by the IPSO 
Step 4: Update the individual and global best-known values.

If the current fitness of a particle is better than the individual best-known fitness $P_{\text {best }}$, then $P_{\text {best }}$ should be replaced with the current fitness; If the best fitness among all particles is better than the global best-known fitness $G_{\text {best, }}$ then $G_{\text {best }}$ should be replaced with that value.

Step 5: Update the velocity and position of each particle.

The velocity and position of each particle should be updated by the said formulas, and the velocity should be constrained in a reasonable interval.

Step 6: Terminate the algorithm and output the optimal network.

According to the termination conditions, the algorithm should be terminated to output the optimal solution, if the maximum number of iterations is reached or the training error falls below a specified level.

Figure 3 explains the flow of the optimization of the BPNN by the IPSO.

\subsubsection{Decision-level fusion based on D-S theory of evidence}

In the D-S theory of evidence [24-27], the set of all possible propositions is defined as a universe $X=\left\{x_{1}, x_{2}, \ldots, x_{n}\right\}$, and the basic belief assignment function is defined as $m: 2^{X} \rightarrow[0,1]$, which satisfies:

$$
\left\{\begin{array}{l}
\mathrm{m}(\phi)=0 \\
0 \leq \mathrm{m}(\mathrm{A}) \leq 1, \forall \mathrm{A} \subset \mathrm{x} \\
\sum_{\mathrm{A} \subset X} \mathrm{~m}(\mathrm{~A})=1
\end{array}\right.
$$

Each subset of propositions is bounded by two values, belief $(B e l)$ and plausibility $(p l)$ :

$$
\begin{gathered}
\operatorname{Bel}(A)=\sum_{B \subseteq A} m(B), \forall A \subseteq X \\
p l(A)=1-\operatorname{Bel}(A)=\sum_{B \cap A \neq \phi} m(B), \forall A \subseteq X
\end{gathered}
$$

Suppose there are two belief functions $\mathrm{Bel}_{1}$ and $\mathrm{Bel}_{2}$ in the same universe. The basic belief assignments of the two belief functions are respectively $m_{1}$ and $m_{2}$, while the focal elements of the two functions are $A_{1}, A_{2}, \ldots, A_{k}$ and $B_{1}, B_{2}, \ldots, B_{n}$, respectively.

If $\sum_{A_{i} \cap B_{j}=\phi} m_{1}\left(A_{i}\right) m_{2}\left(B_{j}\right)<1$, then the combined basic belief assignment $m: 2^{X} \rightarrow[0,1]$ can be expressed as:

$$
m(A)=\left\{\begin{array}{l}
0, A=\Phi \\
\sum_{A_{i} \cap B_{j}=A} \frac{m_{1}\left(A_{i}\right) m_{2}\left(B_{j}\right)}{1-\sum_{A_{i} \cap B_{j}=\phi} m_{1}\left(A_{i}\right) m_{2}\left(B_{j}\right)}, A \neq \Phi
\end{array}\right.
$$

The intersection of the cores of $\mathrm{Bel}_{1}$ and $\mathrm{Bel}_{2}$ is the core of the combined basic belief assignment. The direct sum of $\mathrm{Bel}_{l}$ and $\mathrm{Bel}_{2}$ is denoted as $\mathrm{Bel}_{1} \oplus \mathrm{Bel}_{2}$. Suppose there are multiple belief functions $\mathrm{Bel}_{1}, \mathrm{Bel}_{2}, \ldots, \mathrm{Bel}_{n}$ in the universe $X$, whose basic belief assignments are $m_{1}, m_{2}, \ldots, m_{n}$. If there exits $\mathrm{Bel}_{1} \oplus \mathrm{Bel}_{2} \oplus \cdots \oplus \mathrm{Bel}_{n}$, then the combination of the $n$ belief functions can be expressed as:

$$
\mathrm{Bel}=\left\{\left[\left(\mathrm{Bel}_{1} \oplus \mathrm{Bel}_{2}\right) \oplus \mathrm{Bel}_{3}\right] \oplus \ldots\right\} \oplus \mathrm{Bel}_{n}
$$

\section{SIMULATION AND RESULTS ANALYSIS}

\subsection{Simulation of BPNN optimized by IPSO}

Before the simulation, the parameters of the BPNN and the IPSO were configured as follows: size of particle swarm, $N=20$; number of dimensions of each particle, 2; learning factors, $c_{1}=1.5$ and $c_{2}=1.7$; the maximum number of iterations, 300; the desired error of training, 0.00001 (MSE); number of hidden layer neurons, 8 . The number of hidden layer neurons was determined through several training experiments. The BPNN structure is 6-8-5, i.e. six input layer neurons, 8 hidden layer neurons and 5 output layer neurons. Then, both the BPNN and the BPNN optimized by the IPSO were simulated. The results in Figures 4 and 5 show that the optimized BPNN achieved much more stable fitting results than the original BPNN, which relies on empirical values or expert scoring. Therefore, the local minimums are effectively avoided through the iterative optimization of the BPNN by the IPSO.

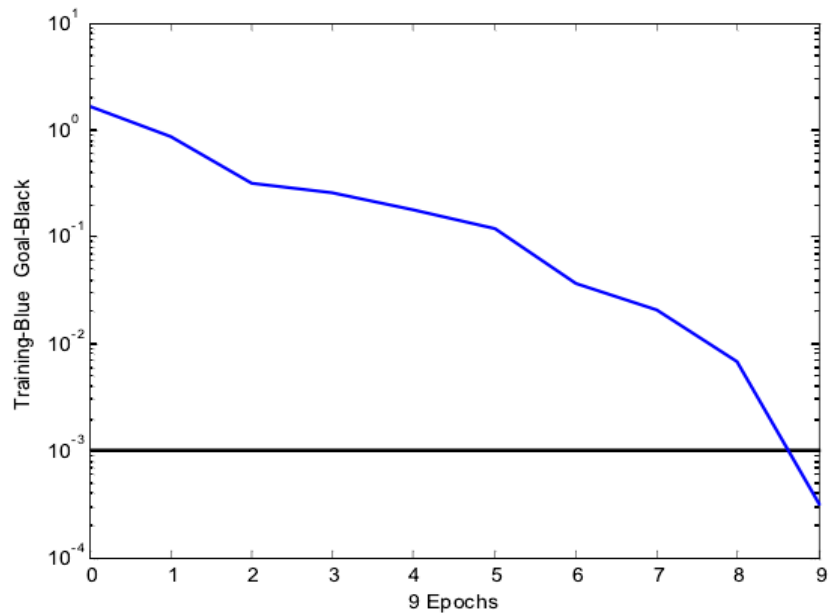

Figure 4. The fitting curve of the original BPNN

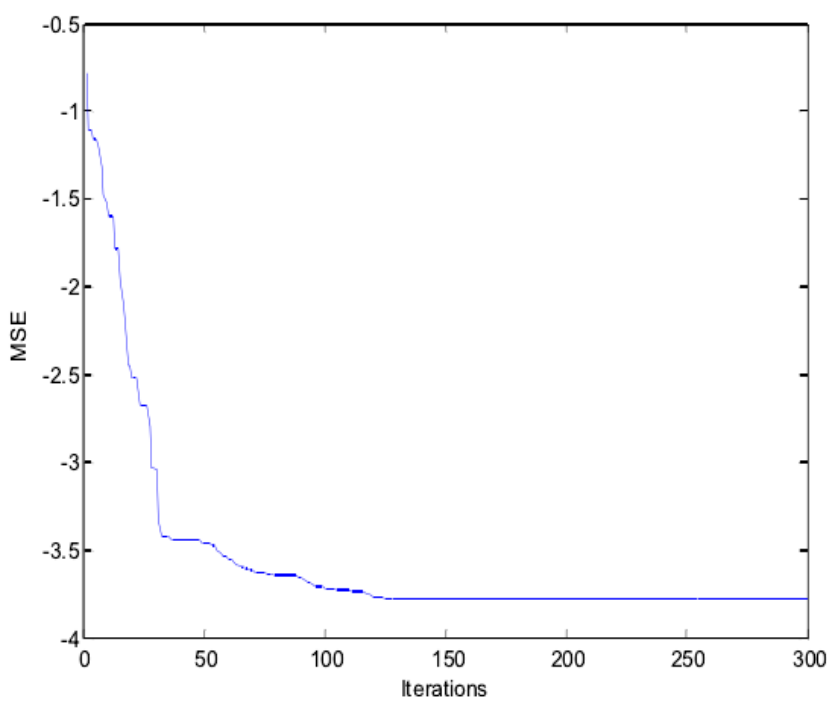

Figure 5. The training error curve of the BPNN optimized by the IPSO 


\subsection{D-S-based decision-making}

The outputs of the optimized BPNN were normalized and taken as the basic belief assignments for the D-S theory of evidence. The evidence set was divided into several unrelated parts. Then, two deductions were carried out based on the D-S theory of evidence: data fusion in the time domain (the information collected at different time from the same location was fused to improve the diagnosis accuracy for that location), and then data fusion in the spatial domain (the identification results under the same state from different locations were fused in the spatial domain to improve the correctness of the final decision).

Two sets of real-time data were collected from four locations, namely, the workface, intake airway, return airway and transport roadway, in a coalmining area of a coalmine in Shanxi Province, China. The collected data were inputted to the optimized BPNN, creating a three-layer network with 6 input layer neurons, eight hidden layer neurons and five output layer neurons. The outputs of the network were taken as the predicted risk of gas outburst. The hazard of gas outburst was divided into five level: safe (level 0), mild (level 1), medium (level 2), severe (level 3) and extremely severe (level 4), denoted as $\left\{F_{1}, F_{2}, F_{3}, F_{4}, F_{5}\right\}$. The predicted level was adopted as the basic belief assignment function for decisionmaking based on D-S theory of evidence. Table 1 shows the values of the basic belief assignment functions.

Then, the information collected at different time from the same location was fused. The results are displayed in Table 2.

As shown in Table 2, the information in the workface and the intake airway was fused well, indicating that the two locations are safe. However, the basic beliefs of the return airway and transport roadway were not clearly distinguishable, especially in the return airway (two levels of risk were obtained here: mild and medium). This calls for further fusion in the spatial domain. Thus, the results under the same state from different locations were fused. The fusion results are shown in Table 3 below.

Through the fusion in the spatial domain, the result shows that the coalmine has a mild risk of gas outburst. In this case, the uncertain is approximately zero.

Table 1. The values of the basic belief assignment functions

\begin{tabular}{ccccccc}
\hline \multirow{2}{*}{ Location } & \multicolumn{5}{c}{ Basic belief assignment functions } \\
& $F_{1}$ & $F_{2}$ & $F_{3}$ & $F_{4}$ & $F_{5}$ & Uncertainty \\
\hline \multirow{2}{*}{1} & 0.987301 & 0.004380 & 0.008124 & 0.998905 & 0.007823 & 0.006729 \\
& 0.981257 & 0.008764 & 0.004231 & 0.973218 & 0.006237 & 0.003219 \\
2 & 0.007691 & 0.983785 & 0.003481 & 0.006372 & 0.998733 & 0.004623 \\
& 0.003789 & 0.989836 & 0.006341 & 0.004125 & 0.975479 & 0.005432 \\
\multirow{2}{*}{3} & 0.003817 & 0.004870 & 0.993872 & 0.005698 & 0.006321 & 0.008726 \\
& 0.003287 & 0.005128 & 0.977863 & 0.002437 & 0.004328 & 0.006584 \\
4 & 0.007612 & 0.798321 & 0.003218 & 0.851283 & 0.007987 & 0.007983 \\
& 0.005436 & 0.842318 & 0.004356 & 0.821872 & 0.008794 & 0.009546 \\
\hline
\end{tabular}

Table 2. The results of data fusion in the time domain

\begin{tabular}{ccccccc}
\hline \multirow{2}{*}{ Location } & \multicolumn{5}{c}{ Basic belief assignment functions } \\
& $F_{1}$ & $F_{2}$ & $F_{3}$ & $F_{4}$ & $F_{5}$ & Uncertainty \\
\hline 1 & 0.005436 & 0.842318 & 0.004356 & 0.006231 & 0.009987 & 0.000954 \\
2 & 0.006231 & 0.832183 & 0.009987 & 0.003219 & 0.007612 & 0.000712 \\
3 & 0.004328 & 0.007634 & 0.821981 & 0.003298 & 0.006326 & 0.001235 \\
4 & 0.005431 & 0.005348 & 0.812768 & 0.003214 & 0.004321 & 0.002543 \\
\hline
\end{tabular}

Table 3. The results of data fusion in the spatial domain

\begin{tabular}{|c|c|c|c|c|c|c|}
\hline \multicolumn{6}{|c|}{ Basic belief assignment functions } & \multirow{2}{*}{ Result } \\
\hline$F_{1}$ & $F_{2}$ & $F_{3}$ & $F_{4}$ & $F_{5}$ & Unce & \\
\hline 0.00001 & 0.43652 & 0.0001 & 0.0000 & 0.0000 & 0.0000 & $F_{2}$ \\
\hline
\end{tabular}

\section{CONCLUSIONS}

The gas outburst in the coalmine is affected by various complex factors. It is difficult to predict the hazard by a single nonlinear method. To solve the problem, this paper combines data mining with information fusion to predict the level of gas outburst of coalmines. The combined method overcomes the difficulty in modelling of information fusion systems, and improves the system's prediction accuracy of gas outburst. The main conclusions are as follows:

(1) Considering the ability of data mining in self-learning modelling, the author established an identification model for gas outburst information based on the BPNN, and optimized the weights and parameters of the BPNN by the IPSO. The optimization enhances the global search ability and convergence speed of the entire system.

(2) The D-S theory of evidence was introduced to fuse the identification results on gas outburst, and make decisions on the gas state of the coalmine. The outputs of the optimized BPNN were adopted as the values of the basic belief assignment functions, eliminating the subjectivity in the assignment process of the D-S theory of evidence. Then, the proposed model was applied the predict the gas outburst in a coalmining area of a coalmine in Shanxi Province, China, using two sets of real-time data collected from the workface, intake airway, return airway and transport roadway. The results show that our model reduced the uncertainty to zero, and improved the accuracy in the prediction of gas outburst in coalmines.

(3) Gas outburst is a hazardous dynamic phenomenon, 
which is influenced and induced by various complex factors. The future research will further explore the formation mechanism of this hazard, and improve the prediction of gas outburst.

\section{ACKNOWLEDGEMENT}

This research is supported by the Special Support Project of Science and Technology Innovation and Entrepreneurship Fund of Tiandi Technology Co., Ltd. (Grant No.: 2018-TDMS017).

\section{REFERENCES}

[1] Nie, B.S., He, X.Q., Wang, E.Y., Liu, Z.T., Sa, Z.Y. (2003). Present situation and progress trend of prediction technology of coal and gas outburst. China Safety Science Journal, 13(12): $1-4$. https://doi.org/10.16265/j.cnki.issn10033033.2003.06.012

[2] Aziz, N., Black, D., Ren, T. (2011). Keynote paper mine gas drainage and outburst control in Australian underground coal mines. Procedia Engineering, 26: 8492. https://doi.org/10.1016/j.proeng.2011.11.2143

[3] Lama, R.D., Bodziony, J. (1998). Management of outburst in underground coal mines. International Journal of Coal Geology, 35(1-4): 83-115. https://doi.org/10.1016/S0166-5162(97)00037-2

[4] Fisne, A., Esen, O. (2014). Coal and gas outburst hazard in Zonguldak Coal Basin of Turkey, and association with geological parameters. Natural Hazards, 74(3): 13631390. https://doi.org/10.1007/s11069-014-1246-9

[5] Blanch, M. (2017). Outburst thresholds-misconceptions, criticisms and context. In: Proceedings of the coal operator's conference. Wollongong: University of Wollongong.

[6] Sobolev, V., Rudakov, D., Stefanovych, L., Jach, K. (2017). Physical and mathematical modelling of the conditions of coal and gas outbursts. Mining of Mineral Deposits, 11(3): 40-49.

[7] Black, D.J. (2019). Review of coal and gas outburst in Australian underground coal mines. International Journal of Mining Science and Technology, 29(6): 815-824. https://doi.org/10.1016/j.ijmst.2019.01.007

[8] An, F., Yuan, Y., Chen, X., Li, Z., Li, L. (2019). Expansion energy of coal gas for the initiation of coal and gas outbursts. Fuel, 235: 551-557. https://doi.org/10.1016/j.fuel.2018.07.132

[9] Kursunoglu, N., Onder, M. (2019). Application of structural equation modeling to evaluate coal and gas outbursts. Tunnelling and Underground Space Technology, 88, 63-72. https://doi.org/10.1016/j.tust.2019.02.017

[10] Fan, C.J., Li, S., Luo, M.K., Du, W.Z., Yang, Z.H. (2017). Coal and gas outburst dynamic system. International Journal of Mining Science and Technology, 27(1): 49-55.

[11] Liu, H.B., Li, Y.B., Wang, F.Z. (2016). Evaluation strategy of gas outburst based on fuzzy neural network and evidence theory. Journal of University of Shanghai for Science and Technology, 3(2): 168-171. https://doi.org/10.13255/j.cnki.jusst.2016.02.012

[12] Tu, Q., Cheng, Y., Guo, P., Jiang, J., Wang, L., Zhang, R.
(2016). Experimental study of coal and gas outbursts related to gas-enriched areas. Rock Mechanics and Rock Engineering, 49(9): 3769-3781. https://doi.org/10.1007/s00603-016-0980-6

[13] Liu, Y.J., Zhao, Q., Hao, W.L. (2015). Study of gas concentration prediction based on genetic algorithm and optimizing BP neural network. Mining Safety \& Environmental Protection, 42(2): 56-60. https://doi.org/10.3969/j.issn.1008-4495.2015.02.014

[14] Papalexakis, E.E., Faloutsos, C., Sidiropoulos, N.D. (2017). Tensors for data mining and data fusion: Models, applications, and scalable algorithms. ACM Transactions on Intelligent Systems and Technology (TIST), 8(2): 16. https://doi.org/10.1145/2915921

[15] Tu, N., Yan, X., Wu, S., Zhu, Y. (2019). Study on earlywarning of coal and gas outburst based on wavelet packet entropy and data fusion. In 2018 7th International Conference on Sustainable Energy and Environment Engineering (ICSEEE 2018). Atlantis Press, 26(4): 759764. https://doi.org/10.14107/j.cnki.kzgc.20180299

[16] Sobczyk, J. (2011). The influence of sorption processes on gas stresses leading to the coal and gas outburst in the laboratory conditions. Fuel, 90(3): 1018-1023. https://doi.org/10.1016/j.fuel.2010.11.004

[17] Grychowski, T. (2014). Multi sensor fire hazard monitoring in underground coal mine based on fuzzy inference system. Journal of Intelligent \& Fuzzy Systems, 26(1): 345-351. https://doi.org/10.3233/IFS-120743

[18] Wu, Y., Gao, R., Yang, J. (2020). Prediction of coal and gas outburst: A method based on the BP neural network optimized by GASA. Process Safety and Environmental Protection, 133: 64-72.

[19] Ruilin, Z., Lowndes, I.S. (2010). The application of a coupled artificial neural network and fault tree analysis model to predict coal and gas outbursts. International Journal of Coal Geology, 84(2): 141-152. https://doi.org/10.1016/j.coal.2010.09.004

[20] Rudakov, D., Sobolev, V. (2019). A mathematical model of gas flow during coal outburst initiation. International Journal of Mining Science and Technology, 29(5): 791796. https://doi.org/10.1016/j.ijmst.2019.02.002

[21] Zhi, S., Elsworth, D. (2016). The role of gas desorption on gas outbursts in underground mining of coal. Geomechanics and Geophysics for Geo-Energy and GeoResources, 2(3): https://doi.org/10.1007/s40948-016-0026-2

[22] Esen, O., Fisne, A., Ökten, G., Black, D.J. (2018). Gas desorption rate of coal seams in Zonguldak coal basic as an indicator of outburst proneness. University of Wollongong Research Online, Coal Operators' Conference, 264-272.

[23] Huang, L., Du, W.W., Ding, L.X. (2012). Particle swarm optimization algorithm based on adaptive Sigmoid inertia weight. Application Research of Computers, 29(1): 32-34. https://doi.org/10.3969/j.issn.10013695.2012.01.008

[24] Geng, J.B., Xu, J., Nie, W., Peng, S.J., Zhang, C.L., Luo, X.H. (2017). Regression analysis of major parameters affecting the intensity of coal and gas outburst in laboratory. International Journal of Mining Science and Technology, 27(2): 327-332.

[25] Wang, L., Liu, S., Cheng, Y.P., Yin, G.Z., Guo, P.K., Mou, J.H. (2017). The effects of magma intrusion on localized stress distribution and its implications for coal mine 
outburst hazards. Engineering Geology, 218: 12-21. https://doi.org/10.1016/j.enggeo.2017.01.002

[26] Yuan, Q., Jia, S., Yuan, Q., Li, Z., Yin, X. (2018). Key process and quality characteristic identification for manufacturing systems using dynamic weighting function and ds evidence theory. International Journal of
Performability Engineering, 14(8): 1651-1665.

[27] Shadrin, A., Diyuk, Y. (2019). Geophysical criterion of pre-outburst coal outsqueezing from the face space into the working. International Journal of Mining Science and Technology, 29(3): 499-506. 